\title{
Effect of Shaft Frequency on Cavitation in a Journal Bearing for Noncentered Circular Whirl
}

David E. Brewe

Propulsion Directorate

U.S. Army Aviation Research and Technology Activity-AVSCOM

Lewis Research Center

Cleveland, Ohio

and

M.M. Khonsari

Ohio State University

Columbus, Ohio

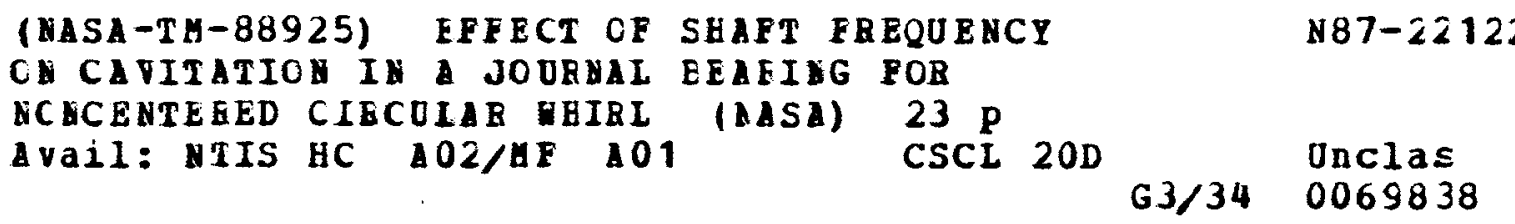

Prepared for the

1987 Annual Meeting of the American Society of Lubrication Engineers Anaheim, California, May 11-14, 1987 


\title{
EFFECT OF SHAFT FREQUENCY ON CAVITATION IN A JOURNAL
}

BEARING FOR NONCENTERED CIRCULAR WHIRL

\author{
David E. Brewe \\ Propulsion Directorate \\ U.S. Army Aviation Research and Technology Activity - AVSCOM \\ Lewis Research Center \\ Cleveland, Ohio 44135 \\ and \\ M.M. Khonsari \\ Ohio State University \\ Columbus, Ohio 43210
}

Abstract

This paper deals with the effect of shaft frequency on the performance of a submerged journal undergoing noncentered circular whirl. The main emphasis of the paper is on the behavior of the vapor cavitation bubble and its effect on the bearing performance as a function of frequency. A cavitation algorithm due to Elrod was implemented in a computer program which solves a time-dependent Reynolds equation. This algorithm automatically handles the boundary conditions by using a switch function and a control volume approach which conserves mass throughout the entire flow. The shaft frequencies in this investigation ranged from $0 \mathrm{rad} / \mathrm{s}$ (squeeze-film damper) to $-104 \mathrm{rad} / \mathrm{s}$ (a case in which oil-whip condition was produced momentarily). For the particular vibration amplitude chosen in this investigation it was observed that vapor cavitation had an effect on the load components for the full range of shaft frequencies investigated.

Nomenc lature

D shaft diameter, m

e eccentricity, m

e dynamic eccentricity, m

$e_{s} \quad$ static eccentricity, m 


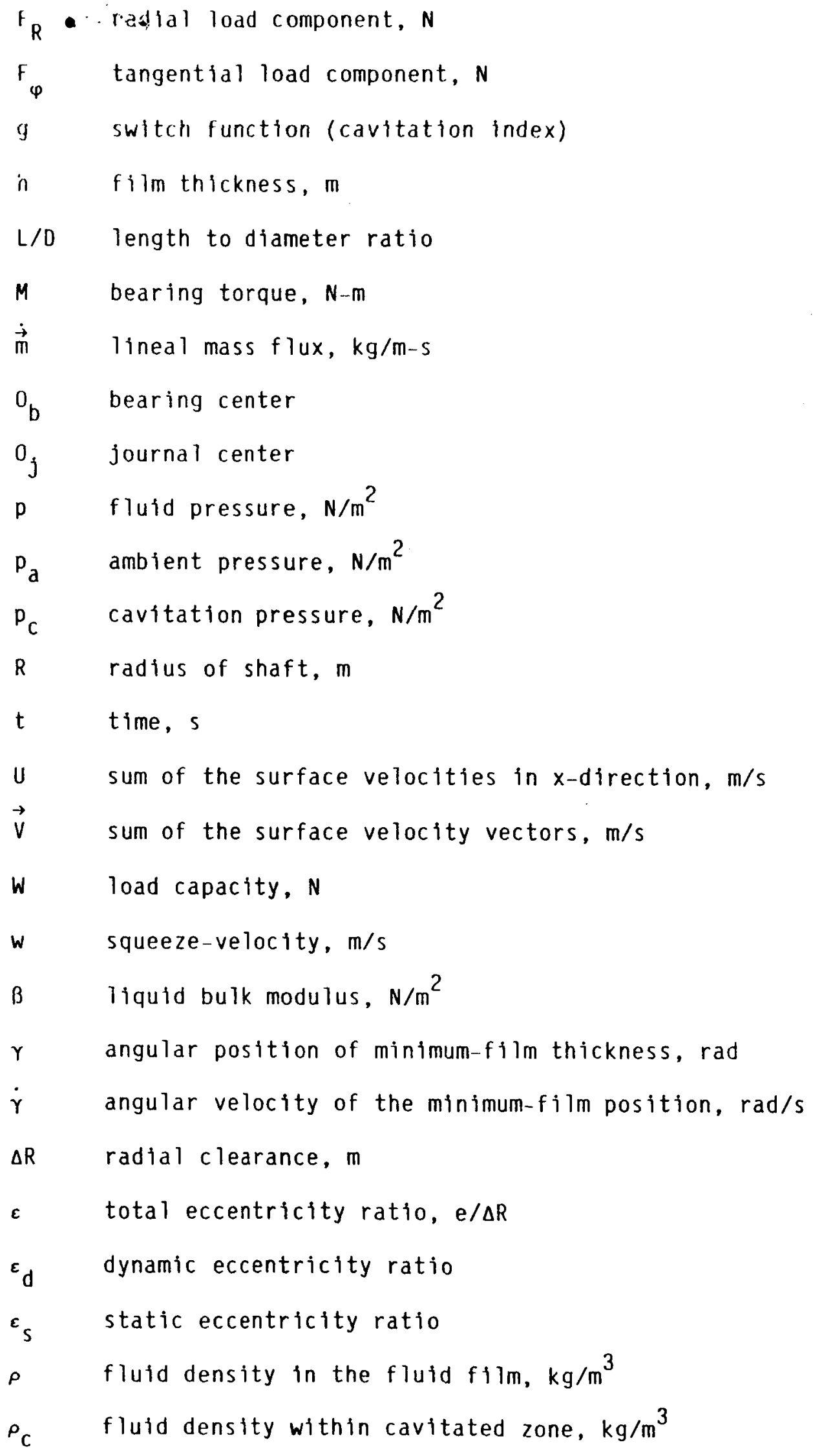


$\theta$ fractional film content in cavitation zone, density ratio $\left(\rho / \rho_{C}\right)$ in full-film zone

$\mu \quad$ dynamic viscosity, $N-5 / m$

$\tau_{s}$ period for 1 revolution of the journal about its own center, $2 \pi / \omega_{5}, s$

Id period for 1 revolution of the journal center about a fixed point relative to housing center, $2 \pi / \omega_{d}, s$

$\varphi \quad$ angular coordinate relative to minimum-film line, rad

$\Psi \quad$ attitude angle, $\tan ^{-1}\left(F_{\varphi} / F_{R}\right)$, rad

$\omega_{d} \quad$ orbital angular velocity of journal center about a fixed point relative to housing center, rad/s

$\omega_{s}$ angular velocity of journal about its own center, $\mathrm{rad} / \mathrm{s}$

\section{INTROOUCTION}

This paper deals with the effect of shaft frequency on the performance of a dynamically loaded journal bearing with vapor cavitation. Despite the importance of cavitation in dynamically loaded bearings, very little work has been reported which fully accounts for the formation of vapor cavitation. Because of the complexity and the computational requirements of the process, most researchers are compelled to assume that in a dynamically loaded bearing the boundary conditions remain similar to a steadily loaded journal bearing (1). An earlier paper by Brewe (2) illustrated some of the consequences of not taking film reformation and the moving boundary into account.

Cavitation can manifest itself in two distinct forms: gaseous cavitation and vaporous cavitation. Gaseous cavitation is generally seen in a steadily loaded journal bearing. It occurs in low pressure regions of the flow where the saturation pressure of the fluid has been reached. This form of cavitation is benign to bearing surface damage (3). In contrast, vaporous cavitation normally occurs in dynamically loaded bearings and is responsible for physical damage to the bearing surfaces as a result of the collapse of the vaporous cavities on the surfaces. Jacobson and Hamrock $(4,5)$ recorded the 
appearance of vapor cavitation in a dynamically loaded journal bearing for the first time by using high-speed photography. More recently, Walton et al. have used high-speed photography to observe cavitation in squeeze-film dampers. ("Experimental Observation of Cavitating Squeeze Film Dampers" presented at the 1986 ASME/ASLE Tribology Conference, Oct. 20-22, 1986, Pittsburgh, PA. To be published in Journal of Tribology, ASME.)

Further, cavitation plays an important role in the stability of rotating machinery. A previous investigation by Brewe (2) verified that cavitation can generate some load capacity and should not be taken lightly in theoretical studies. Therefore, it is important to further study the effect of cavitation on the performance of the bearing.

This study centers on the theoretical investigation of the performance of the bearing with particular attention to cavitation effects. The cavitation algorithm used is based on the mass conservation throughout the entire flow region.

THEORY

Cavitation Algorithm

A computer program was developed based on Elrod's algorithm ( 6 ) that combines a control volume approach to deriving the finite-difference equation with a switching function that automatically handles the moving boundary condition at the liquid-vapor interface. The complication in satisfying the boundary condition is avoided by defining a single independent parameter $\theta$ which links the full-film region and the cavitated region. This parameter is defined by the ratio of the local density to that of the cavitation density, i.e.,

$$
\theta=\frac{e}{\rho_{C}}
$$


It follows that in the cavitation region $\theta<1.0$ and in the full-film region $\theta>1.0$. A switch function $g$ was defined as "on" $(g=1)$ and "off" ( $g=0)$ in the full-film and cavitation regions respectively. By using this property, a universal Reynolds equation was defined as indicated:

$$
\frac{\partial(\rho h)}{\partial t}+\vec{\nabla} \cdot \dot{\vec{m}}=0
$$

where $\vec{m}$ represents the lineal mass flux and is given by

$$
\frac{\vec{m}}{\rho_{C}}=\frac{\theta h \vec{v}}{2}-\frac{h^{3}}{12 \mu} \beta g(\theta) \vec{\nabla} \theta
$$

In the cavitation region, the second term vanishes since $g=0$. This equation was integrated in time by using an Euler's method to advance in time. At every time step the difference equation was solved by an alternating direction implicit (ADI) scheme. The switch function was updated after each half-time step. Occasionally, during the collapse of the vapor bubble, liquid was piling up at the boundary. This was due to an inherent time lag of the switch function with the calculation. Consequentiy, the switch function was immediately updated to accommodate the movement of the boundary, and the calculation was reiterated.

The results of the program developed were authenticated by comparison to an experiment of Ref. (4). When compared to the pseudo-Gümbel boundary conditions (2), the results predicted a significant difference in the computed load capacity (up to 20 percent). Furthermore, it was found that the size, life, and location of the cavitation bubble were considerably affected by imposing the pseudo-Gümbel boundary conditions. 
Fluid Velocity Under Dynamic Loading

Consider the bearing geometry shown in fig. 1. As shown, the minimum-film thickness rotates clockwise around the bearing at a whirl frequency of $y$. Jacobson and Hamrock (5) show that from this diagram $Y$ can be evaluated

$$
r=\tan ^{-1}\left[\frac{\varepsilon_{d} \sin \left(w_{d} t\right)}{\varepsilon_{s}+\varepsilon_{d} \cos \left(w_{d} t\right)}\right]
$$

so that

$$
\dot{\gamma}=\frac{\varepsilon_{d}\left[\varepsilon_{s} \cos \left(w_{d} t\right)+\varepsilon_{d}\right] \omega_{d}}{\varepsilon_{s}^{2}+\varepsilon_{d}^{2}+2 \varepsilon_{s} \varepsilon_{d} \cos \left(w_{d} t\right)}
$$

The total eccentricity e defined as the instantaneous distance between $0_{b}$ and $0_{j}$ is:

$$
e=\frac{e_{d} \sin \left(w_{d} t\right)}{\sin \gamma}
$$

The film thickness is, therefore, defined as follows:

$$
h=\Delta R(1-\varepsilon \cos \varphi)
$$

The sliding velocity of the journal is

$$
U=\left(\omega_{S}-2 \dot{\gamma}\right) R
$$

Test Bearing

The bearing simulated in this study is based on an experimental study of a journal bearing submerged in Dexron lubricant (4). The journal rotated about its own fixed center while the bearing vibrated in a noncentered circular motion keeping the axes of vibration parallel to that of rotation. Note that there are two time scales in this problem: one, $\tau_{S}$, is associated with the spin of the journal about its own center; the other, $\tau_{d}$, is associated with the translational motion of the journal center about a fixed point. The ratio of these time scales $\tau_{d} / \tau_{s}$ is, in some sense, a measure of wedge to squeeze action taking place. This range varied from 0.00 to 1.13 in this study. The data used in the simulation are summarized in Table 1. 
RESULTS ANO DISCUSSION

Effect of Shaft Frequency on Cavitation

Table 2 lists the various shaft frequencies run in this analysis and indicates the effect they had on cavitation. The times listed in the table are relative to the start of the orbit. The period for the noncentered shaft whirl was $67.78 \mathrm{~ms}$ for each case. Cavitation persisted for a longer period of time in the case of the squeeze-film damper (i.e., $48.91 \mathrm{~ms}$ ) than in the other cases listed. The bubble life decreased with increasing shaft frequency. The cavitation desinence (bubble collapse) was weakly affected by shaft frequency. However, as the shaft frequency was increased the cavitation inception was delayed which gave rise to a shorter bubble life.

Orbital Pressure and Cavitation History

Figures 2 and 3 illustrate the responding pressure variation to the motion of the shaft undergoing noncentered circular whirl for two contrasting situations. The case of a squeeze film damper (i.e. $\omega_{s}=0$ ) in Fig. 2 is contrasted with the case in which the oil-whip condition is momentarily observed (i.e. $\omega_{s}=-104.29 \mathrm{rad} / \mathrm{s}$ ) in Fig. 3. Both cases have an orbital frequency of $-92.7 \mathrm{rad} / \mathrm{s}$. In each figure, part (a) illustrates the starting position (i.e. $\varepsilon=0.1$ ). Parts (a) to (f) depict several key events in the clockwise circular motion of the journal center within the bearing.

Figure 2(a) represents the position of the journal within the housing and the associated pressure distribution at the instant that the eccentricity is at a minimum. The dark oblong region indicates the shape and position of a vapor bubble downstream of the minimum-film line. The position of the bubble within the small clearance space of the journal bearing is also indicated. At this particular instant the tendency of the pressure flow along the side of the bubble to cause the bubble to collapse becomes greater since it is proportional to $h^{3}$. The journal center moves in a clockwise orbit from Figs. $2(a)$ to (b) 
(nearly one-eighth of a revolution). While the eccentricity is increasing, the minimum film is decreasing which gives rise to the squeeze action in the vicinity of the minimum-film line. A slight rise in the pressure field can be observed there. Note in Fig. 2(b) that the vapor bubble has collapsed farther downstream from its position in Fig. $2(a)$. It is located closer to the position of maximum-film thickness.

Cavitation is absent from Fig. $2($ b) to (c). In Fig. 2(c), a new cavitation bubble appears, and the pressure generation in the region of the minimum-film line becomes significantly large because of the squeeze and wedge effects taking place there. Figure $2(d)$ represents the configuration of the bearing when the maximum pressure occurs. Note that this happens $5.3 \mathrm{~ms}$ before the maximum eccentricity is reached because of the motion of the shaft [Fig. 4(a)]. As the journal approaches the end of the squeeze cycle and begins to separate from the shaft, the sliding velocity approaches a minimum value and the squeeze velocity passes through zero. The effect of this on the pressures more than offsets any increase in pressure that would have been realized because of the smaller minimum-film thickness.

Figure 2(e) represents the end of the squeeze cycle and the beginning of the separation cycle. Now the minimum film begins to increase which creates a suction effect, an accompanying growth in the bubble, and a dissipation of the pressure hump noted in Fig. $2(f)$. It is interesting to note that the eccentricity is the same $(0.78)$ for figs. $2(d)$ and $(f)$. The drastic difference in the pressure plot is a result of the squeeze velocity having opposite signs in the two situations. The minimum-film thickness continues to increase as it moves in a clockwise direction. The representation shown in Fig. $2(a)$ is repeated as are the representations for each succeeding orbit.

A very significant difference between figs. $2(e)$ and $3(d)$ occurs when the eccentricity is a maximum (i.e. $\boldsymbol{c}=0.80$ ). In the case of the squeeze-film 
damper (i.e. $\omega_{s}=0$ ), Fig. 2(e) illustrates a sizeable pressure buildup and a well developed cavitation bubble. However, for the case in which $\omega_{s}=-104.29 \mathrm{rad} / \mathrm{s}$, Fig. $3(\mathrm{~d})$ shows no pressure buildup or cavitation. This condition arises because the net flow produced by the squeezing and sliding motion is zero. The whirl frequency $\dot{y}$ at this instant is equal to one-half the shaft frequency and is commonly referred to as the "half-frequency whirl" condition.

Load as a Function of Shaft Frequency

Figure $5(a)$ to (d) illustrates how the load-carrying capacity varies as a function of the eccentricity ratio for various shaft frequencies $\omega_{S}$. A case representing a squeeze-film damper $\left(\omega_{s}=0\right)$ is shown in Fig. 5(a). The load variation through one orbit of circular whirl resembles a hysteresis effect which is due to the occurrence of cavitation in the cycle (7). This effect is prevalent for all of the shaft frequencies studied in this investigation.

Each portion of parts (a) and (d) of Fig. 5 is labeled for discussion purposes; the reader should simultaneously refer to the corresponding pressure distributions presented in Figs. 2 and 3 , respectively.

For the particular cases studied, it can be said that increasing the shaft frequency had little effect on the onset of desination (bubble collapse) and the location of the peak load (viz. Fig. 5). However, the magnitude of the peak load decreased with the increase in the shaft frequency.

The variation of the shape of the $W-\varepsilon$ curve with frequency is interesting and well worth discussing. The portion of the cycle beginning with the squeeze motion through the peak pressure [Fig. 5(a) to (d)] is essentially invariant with the shaft frequency. But around the vicinity of $\varepsilon=0.8$ where the separation occurs, the effect of shaft frequency is more apparent. Here the $W-\varepsilon$ develops an increasingly steep gradient with increasing shaft frequency. Finally for the case $\omega_{s}=-104.29 \mathrm{rad} / \mathrm{s}$ 
[Fig. 5(d)] the load capacity vanishes completely when the whirl frequency $(\dot{\gamma})$ is equal to one-half the shaft frequency. This frequency can be predicted by examining equation [7]. The squeeze and sliding velocity for this case is plotted in Fig. $4(\mathrm{C})$.

It may also be noted that on the return cycle (Fig. 5 from point $f$ to a) there is no appreciable load variation where $\omega_{s}=0$. As the frequency increases, the variation in load with eccentricity in that portion increases. This is a noticeable variation (roughly $200 \mathrm{~N}$ ) at the shaft frequency of $-104.29 \mathrm{rad} / \mathrm{s}$. After passing through the load reduction, the sliding and squeeze term in the Reynolds equation recovers to contribute to the load capacity. Another contributing factor to the load capacity in the separation cycle is the cavitation. This can occur when the bubble moves downstream from the minimum-film line so that it positions itself diametrically opposite to the directional load line described by the attitude angle.

Radial and Tangential Loads

Figure 6 illustrates how the shaft frequency can affect the dynamic loading (radial and tangential) as a function of eccentricity through one whirl orbit. Both the radial and tangential loads demonstrate a "hystereticlike" response with eccentricity. This effect is a result of cavitation occurring through some portion of the cycle. [See Refs. (2) and (7).] Operation at the different shaft frequency conditions has a very pronounced effect on the tangential load but only a slight effect on the radial load. As the shaft frequency is increased, the tangential load plots become less hysteretic (See Fig. 6.) in nature. This is mainly because the onset of cavitation has been delayed at the higher shaft frequencies. Further, at the end of the squeeze cycle (i.e. $\varepsilon=0.8$ in this study) the tangential load decreases as the shaft frequency is increased. This is because at this particular stage in the motion the conditions for half-frequency whirl are 
being approached for increasing shaft frequency. When the conditions for half-frequency whirl are met [i.e. in Fig. $6(\mathrm{c})]$, the tangential load and radial load are identically zero.

\section{Attitude Angle}

The effect of the shaft frequency on radial and tangential load is summarized in the attitude angle. The variation of the attitude angle with eccentricity through one circular orbit is shown in Fig. 7. These plots correspond to the case of a squeeze-film damper $\left(\omega_{s}=0\right)$ and the case of synchronous shaft and vibration frequency (i.e. $\omega_{s}=\omega_{d}$ ) respectively. In the damper case [Fig. 7(a)], the asymmetry in the plot is again a result of the cavitation present throughout a portion of the whirl cycle. In Fig. 7(b) the delay of the onset of cavitation diminishes the asymmetry of the plot. Also, the magnitude of the attitude angle remains small (i.e. $\psi \leq 30^{\circ}$ ) throughout most of the cycle (i.e. $0.15 \leq \varepsilon \leq 0.79$ ). At the end of the squeeze cycle the sign is changed, and it very quickly becomes small again for the remainder of the whirl cycle. In this instance the effect of the higher shaft frequency has been to suppress the magnitude of the attitude angle through a large portion of the cycle.

Bearing Torque

The variation of the bearing torque with eccentricity is shown in fig. 8 for three different cases. The bearing torque is a measure of the force required to overcome the friction force in the fluid film [See ref. (2)]. For the squeeze-film damper [Fig. $8(a)$ ], the bearing torque is generally higher throughout the whirl orbit than in any of the cases that included shaft spin. As the shaft frequency increased, the resultant effective siliding velocity decreased (Fig. 4) and, consequentiy, the amount of shear in the fluid. Note that the bearing torque becomes zero for the oil-whip condition [Fig. $8(\mathrm{c})$; $\varepsilon=0.8]$. 
CONCLUSIONS

A study of the effect of shaft frequency on bearing and damper performance under dynamic conditions is presented. The motion considered in this study was that of noncentered circular whirl. The study revealed the following:

1. Increasing the shaft frequency caused a delay in the onset of cavitation. Since the collapse of the bubble occurs at nearly the same time in each case, the life of the bubble is decreased with increasing shaft frequency.

2. During separation (i.e., $\partial h / \partial t>0$ ) increasing shaft frequency results in an appreciable load variation with decreasing eccentricity. This is attributable to the size and location of the cavitation bubble and to the recovery of the wedge and squeeze effects.

3. Operation at the different shaft frequencies has a very pronounced effect on tangential load, but only a slight effect on radial load.

4. At the oil-whip condition it was verified that the bearing lost all load-carrying capacity, and bearing torque was zero.

\section{REFERENCES}

1. Marsh, H., "Cavitation in Dynamically Loaded Journal Bearings," Cavitation and Related Phenomena in Lubrication, edited by D. Dowson, M. Godet, and C.M. Taylor, Mechanical Engineering Publications, 1974, pp 91-95.

2. Brewe, D.E., "Theoretical Modeling of the Vapor Cavitation in Dynamicaliy Loaded Journal Bearings," ‥ Tribology, 108, pp. 628-638 (1986).

3. Wilson, R.W., "Cavitation Damage in Plain Bearings," Cavitation and Related Phenomena in Lubrication, edited by D. Dowson, M. Godet, and C.M. Taylor, Mechanical Engineering Publications, 1974, pp. 177-187. 
4. Jacobson, B.0., Hamrock, B.J., "High-Speed Motion Picture Camera Experiments of Cavitation in Dynamically Loaded Journal Bearings," $\mathrm{J}$. Lubr. Technol., 105, pp. 446-452 (1983).

5. Jacobson, B.0., Hamrock, B.J., "Vapor Cavitation in Dynamically Loaded Journal Bearings," Second International Conference on Cavitation, Mechanical Engineering Publications, 1983, pp. 133-140.

6. Elrod, H.G., "A Cavitation Algorithm," 3. Lubr. Technol., 103, pp. 350-354 (1981).

7. Brewe, D.E., and Jacobson, B.0., "Effect of Vibration Amplitude on Cavitation in Journal Bearings," NASA TM-88826, 1986. (to be published in Wear, 1986)

TABLE 1. - OPERATING CONDITIONS

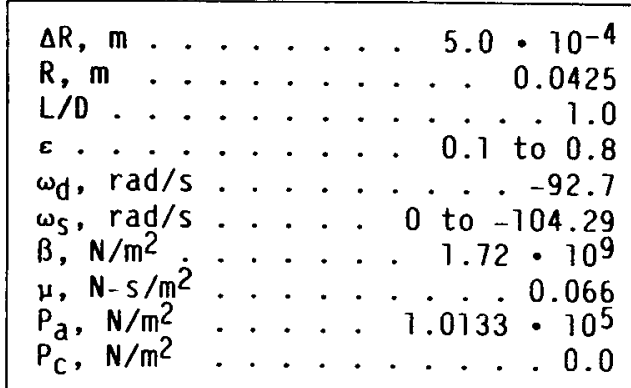

TABLE 2. - EFFECT OF SHAFT FREQUENCY ON CAVITATION

\begin{tabular}{|c|c|c|c|c|c|}
\hline $\begin{array}{c}\text { Shaft } \\
\text { frequency } \\
\text { rad/s }\end{array}$ & \multicolumn{2}{|c|}{ Cavitation inception } & \multicolumn{2}{|c|}{ Cavitation desinence } & $\begin{array}{c}\text { Bubble } \\
\text { i ife, } \\
\text { ms }\end{array}$ \\
\cline { 2 - 6 } & $\begin{array}{c}\text { Time, } \\
\text { ms }\end{array}$ & $\varepsilon$ & $\begin{array}{c}\text { Time, } \\
\text { ms }\end{array}$ & $\varepsilon$ & \\
\hline 0.00 & 24.300 & 0.724 & 73.210 & 0.222 & 48.91 \\
-15.45 & 26.205 & .751 & 73.410 & .228 & 47.21 \\
-30.90 & 28.305 & .774 & 73.610 & .234 & 45.31 \\
-46.35 & 30.305 & .789 & 73.810 & .247 & 43.51 \\
-61.80 & 32.005 & .797 & 74.010 & .248 & 42.01 \\
-77.25 & 33.505 & .800 & 74.110 & .250 & 40.61 \\
-92.70 & 34.505 & .800 & 74.210 & .254 & 39.71 \\
-104.29 & 34.805 & .800 & 73.910 & .243 & 39.11 \\
\hline
\end{tabular}




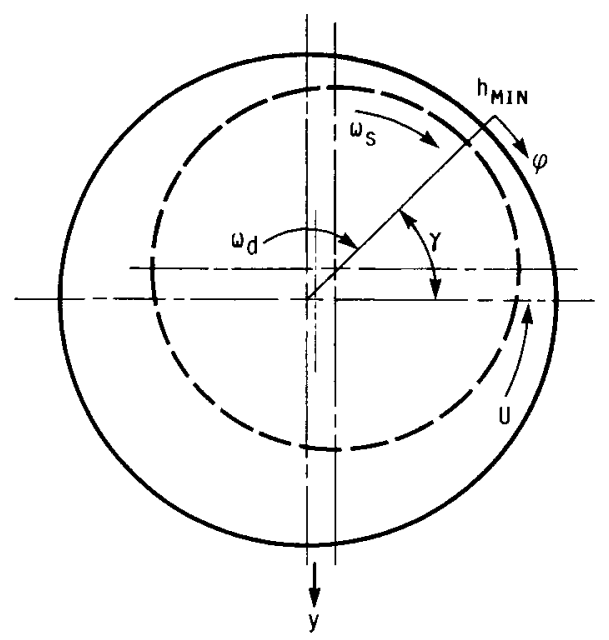

(a) SIDE VIEW.

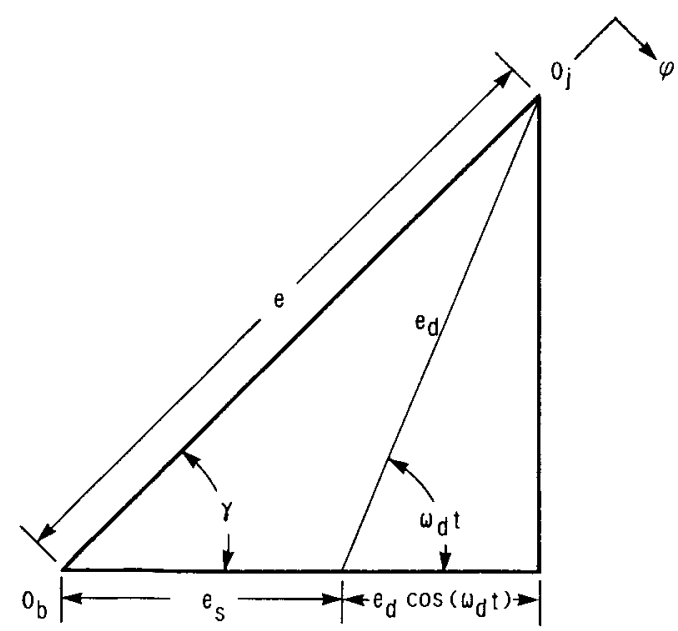

(b) MAGNIFIED VIEW OF RELATIVE CENTERS.

FIG. 1. - JOURNAL BEARING CONFIGURATION. 


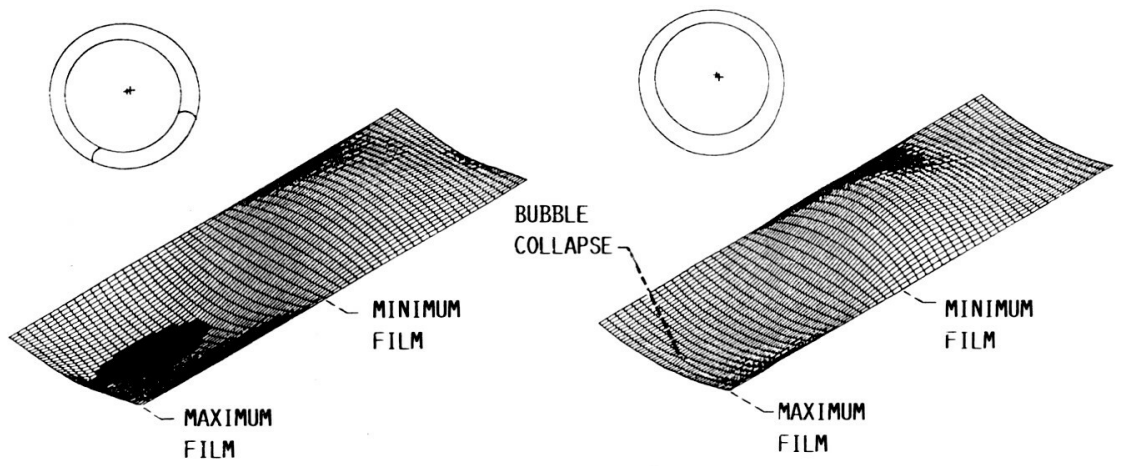

(a) BEGINNING OF SQUEEZE CYCLE. $(t=0$ MS: $\varepsilon=0.1$.

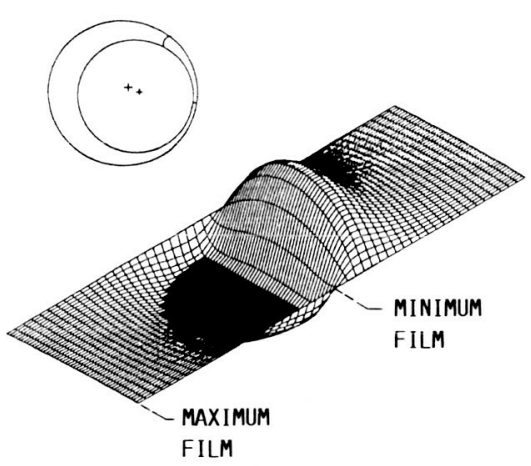

( $f$ ) BUBBLE EXPANSION. $(t=39.31 \mathrm{~ms}$ : $\varepsilon=0.78$. (b) BUBBLE COLLAPSE. $\quad(t=5.43 \mathrm{mS}$ $\varepsilon=0.22$. )

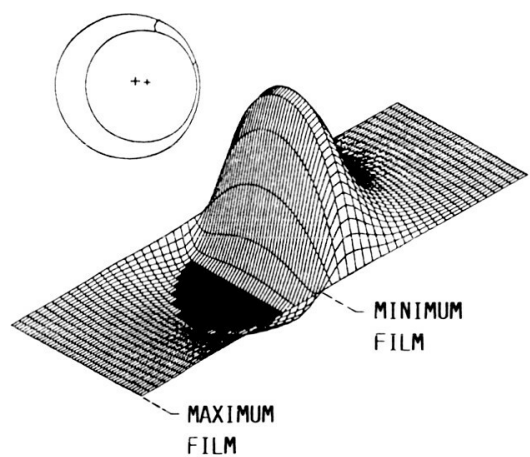

(e) BEGIN SEPARATION. $(\mathrm{t}=33.91 \mathrm{mS}$; $\varepsilon=0.80 .1$

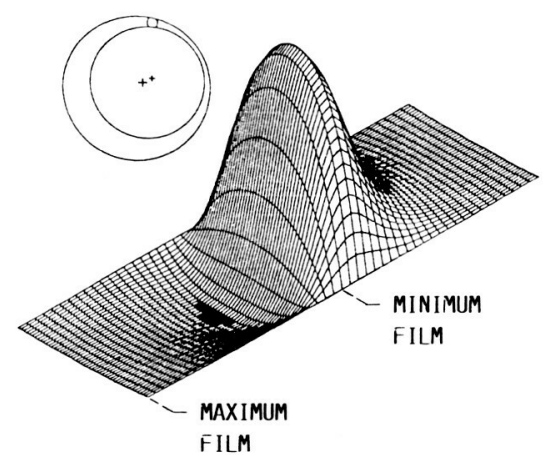

(c) CAVITATION INCEPTION. $(t=24.30 \mathrm{MS}$; $\varepsilon=0.72$.

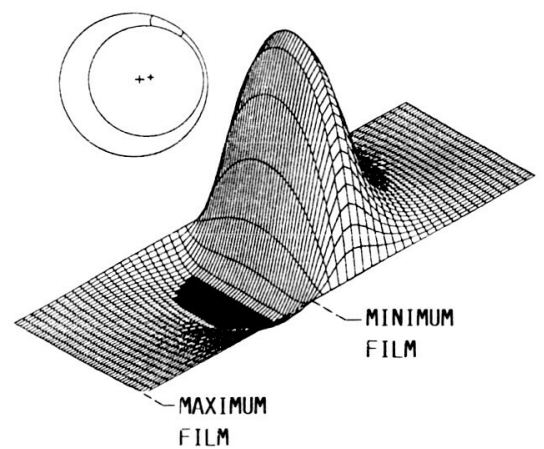

(d) PEAK PRESSURE. $(t=28.61 \mathrm{mS}$; $\varepsilon=0.78$. )

FIG. 2. - PRESSURE DISTRIBUTION AND BEARING CONFIGURATION FOR FULL PERIOD OF SHAFT WHIRL. ( $\omega_{S}=0$ RAD/S. FIGURES (a) TO $(f)$ ARE VIEWED CLOCKWISE IN TIME.) 


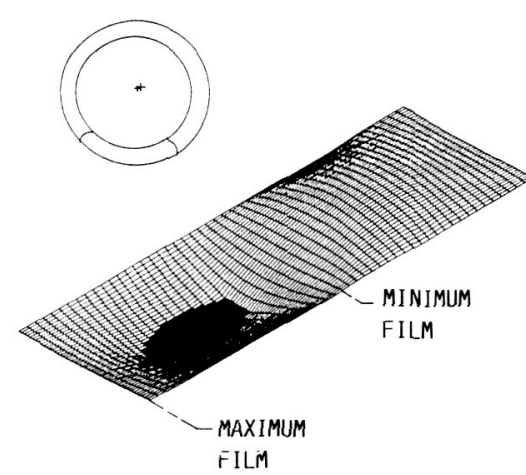

(a) BEGINNING OF SQUEEZE CYCLE. $(\mathrm{t}=0 \mathrm{MS} ; \varepsilon=0.1$.

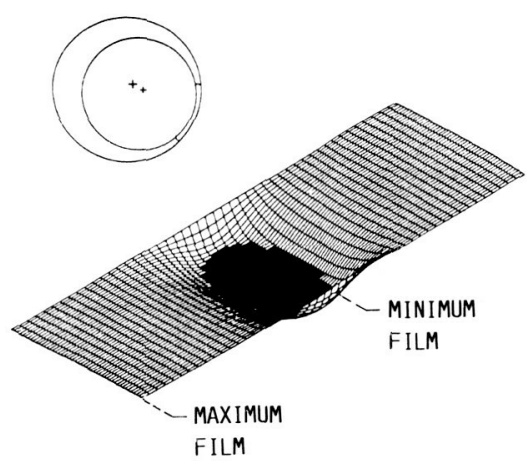

$(f)$ BUBBLE EXPANSION. $(t=4 i .41 \mathrm{MS}$; $\varepsilon=0.75$.)

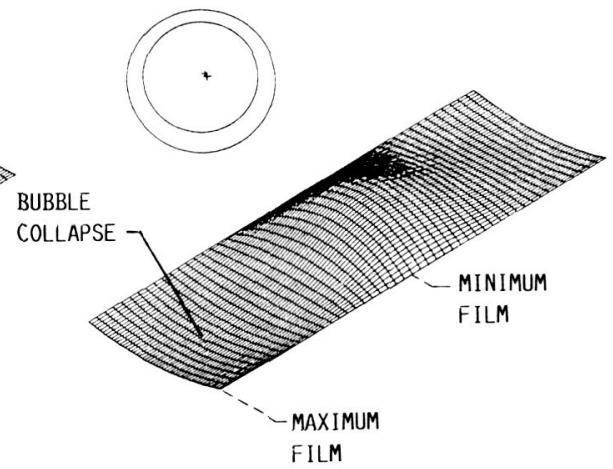

(b) BUBBLE COLLAPSE. ( $t=6.2 \mathrm{Ms}$; $\varepsilon=0.24$.)

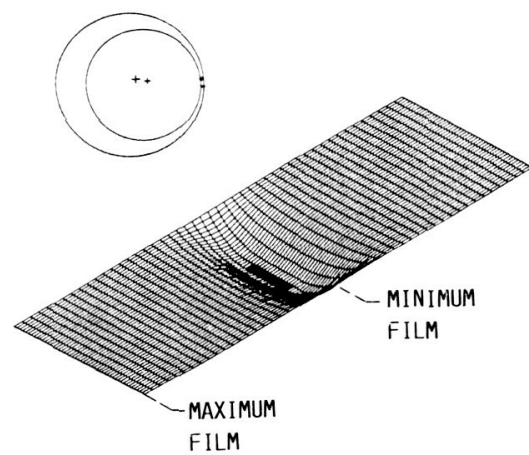

(e) CAVITATION INCEPTION. $(\mathrm{t}=34.81 \mathrm{mS}$ : $\varepsilon=0.799$.)

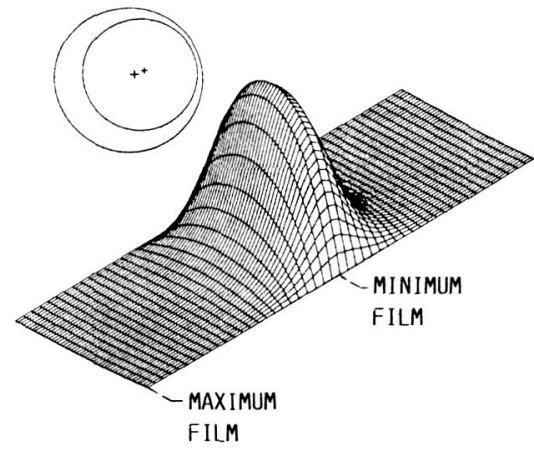

(c) PEAK PRESSURE. $(\mathrm{t}=26.31 \mathrm{MS}$ : $\varepsilon=0.75$.

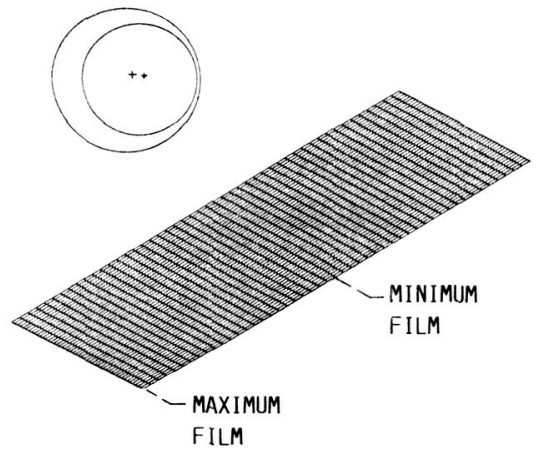

(d) BEGIN SEPARATION OIL-WHIP CONDITION. $(t=33.91$ MS; $\varepsilon=0.80$.

Fig. 3. - PRESSURE distribUtion AND BEARING CONFIGURATION FOR FULL PERIOD OF SHAFT WHIRL. $\left(\omega_{s}=-104.29\right.$ RAD/s; FIGURES (a) TO ( $f$ ) ARE VIEWED CLOCKWISE IN TIME.) 

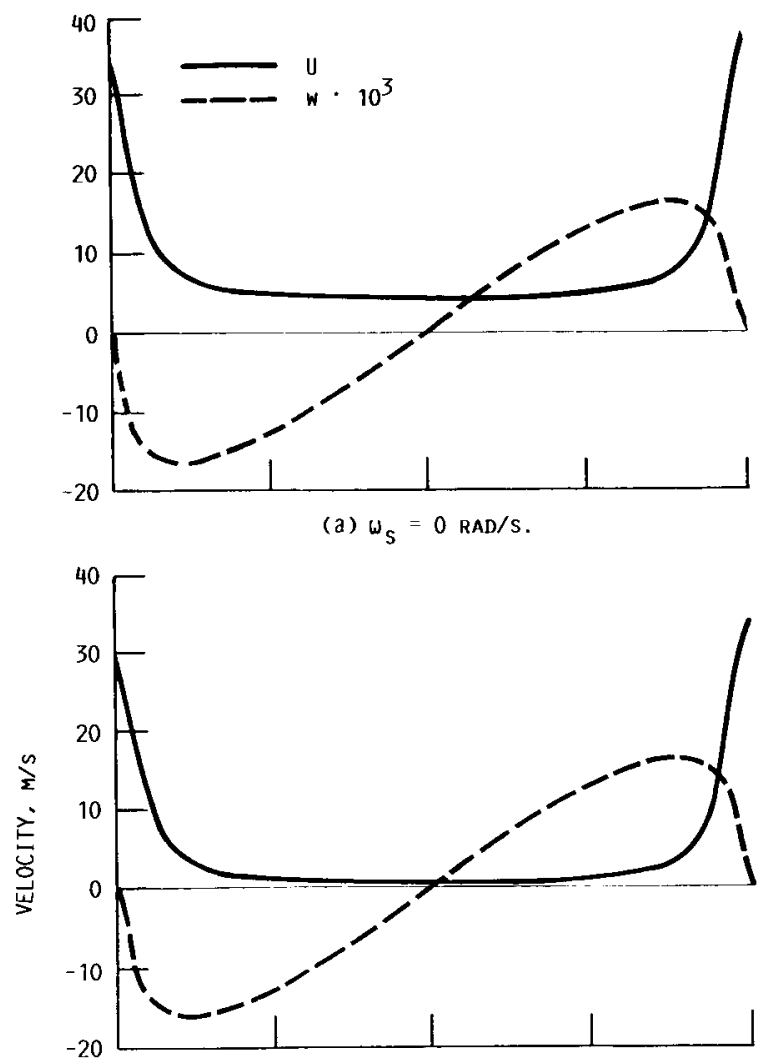

(b) $\omega_{\mathrm{S}}=-92.7 \mathrm{RAD} / \mathrm{s}$.

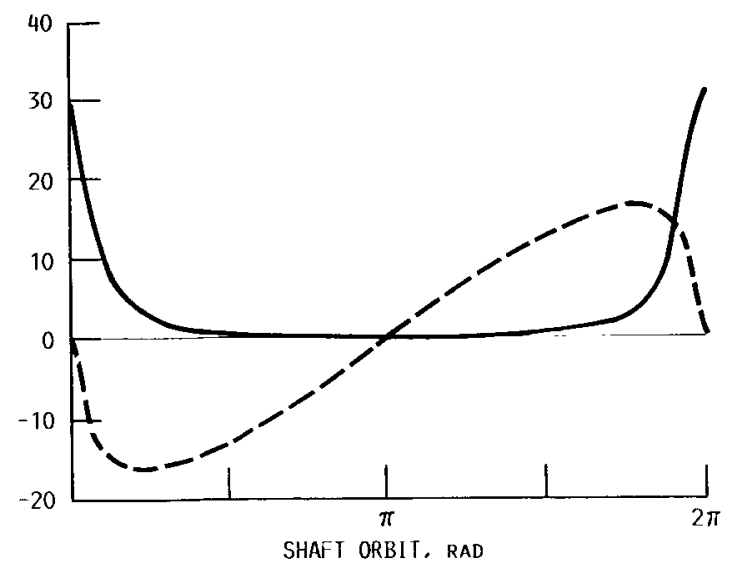

(C) $\omega_{\mathrm{S}}=-104.29 \mathrm{RAD} / \mathrm{s}$.

FIG. 4. - SURFACE VELOCITY SUM (U) AND SQUEEEZE VELOCITY $(W)$ FOR FULL PERIOD OF SHAFT WHIRL AT VARIOUS FREQUENCIES. 


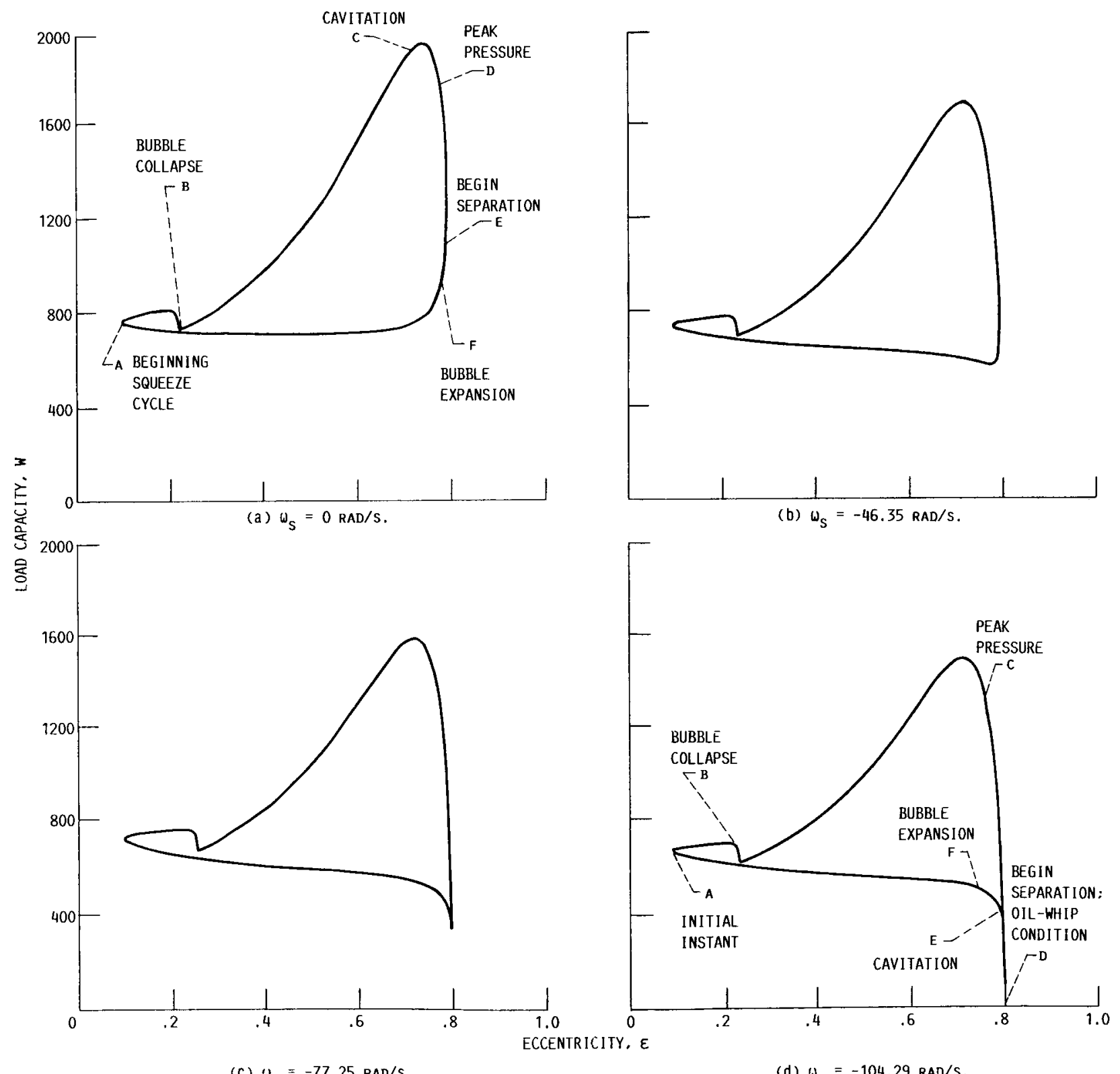

(C) $\omega_{S}=-77.25 \mathrm{RAD} / \mathrm{s}$.

(d) $\omega_{\mathrm{S}}=-104.29 \mathrm{RAD} / \mathrm{S}$.

FIG. 5. - LOAD CAPACITY AS FUNCTION OF ECCENTRICITY RATIO AT FOUR DIFFERENT FREQUENCIES. 

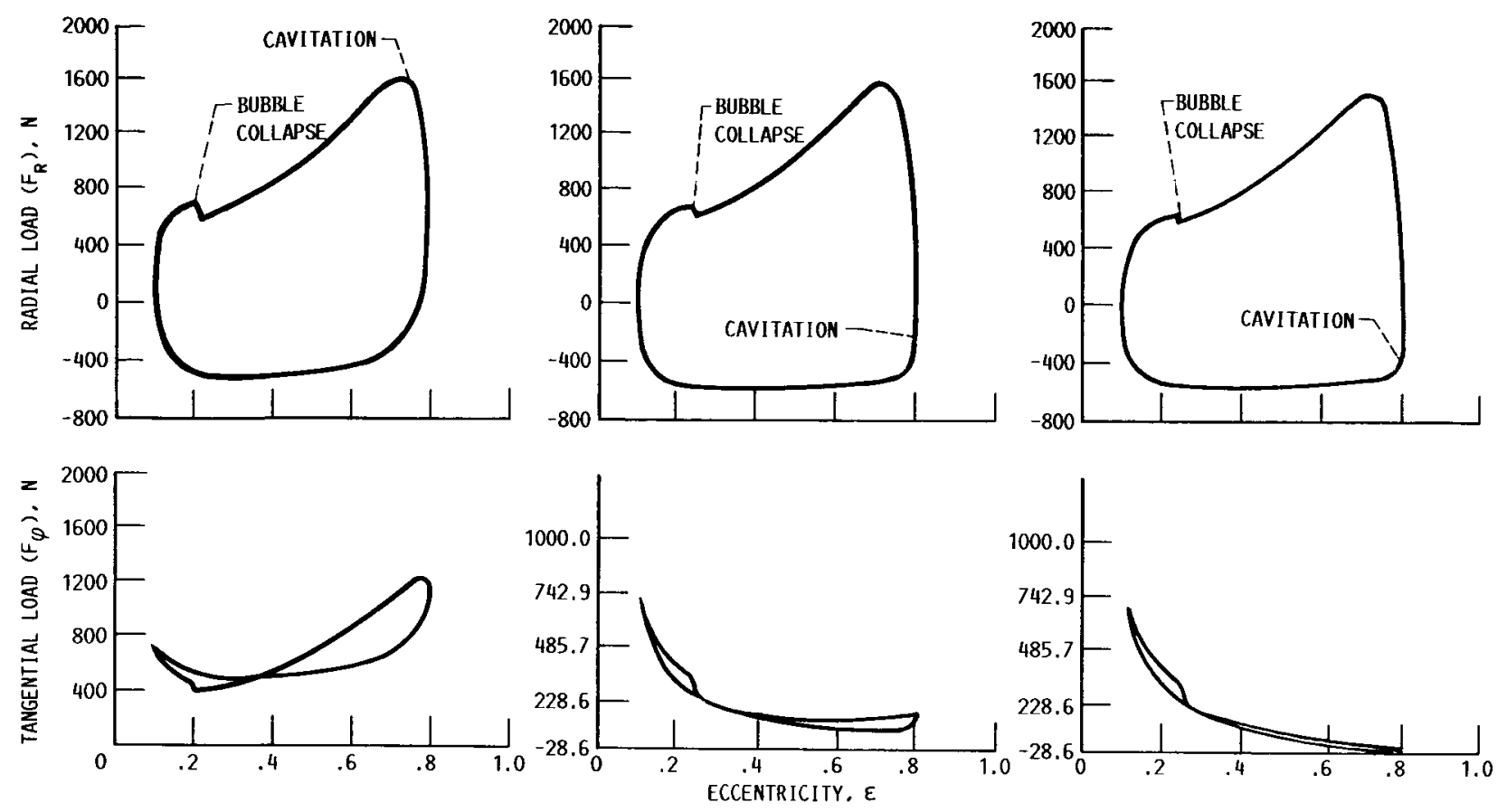

(a) $w_{S}=0 \mathrm{RAD} / \mathrm{s}$.

(b) $\omega_{\mathrm{S}}=-92.7 \mathrm{RAD} / \mathrm{s}$.

(c) $\omega_{S}=-104.29 \mathrm{RAD} / \mathrm{s}$.

Fig. 6. - RADIAL AND tANGENTIAL LOAD AS FUnCtion OF ECCENTRICITY RATIO AT THREE DIFFERENT FREQUENCIES.

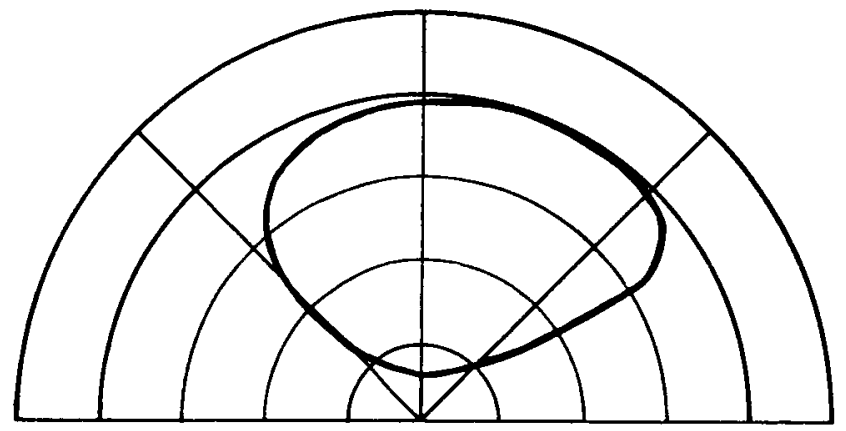

(a) $\omega_{\mathrm{S}}=0 \mathrm{RAD} / \mathrm{s}$.

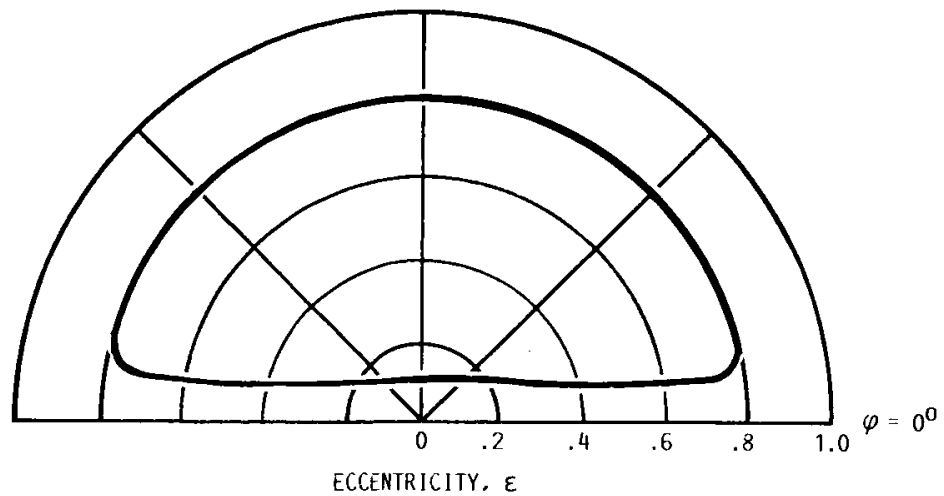

(b) $\omega_{S}=-92.7 \mathrm{RAD} / \mathrm{s}$.

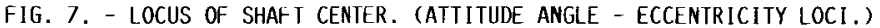



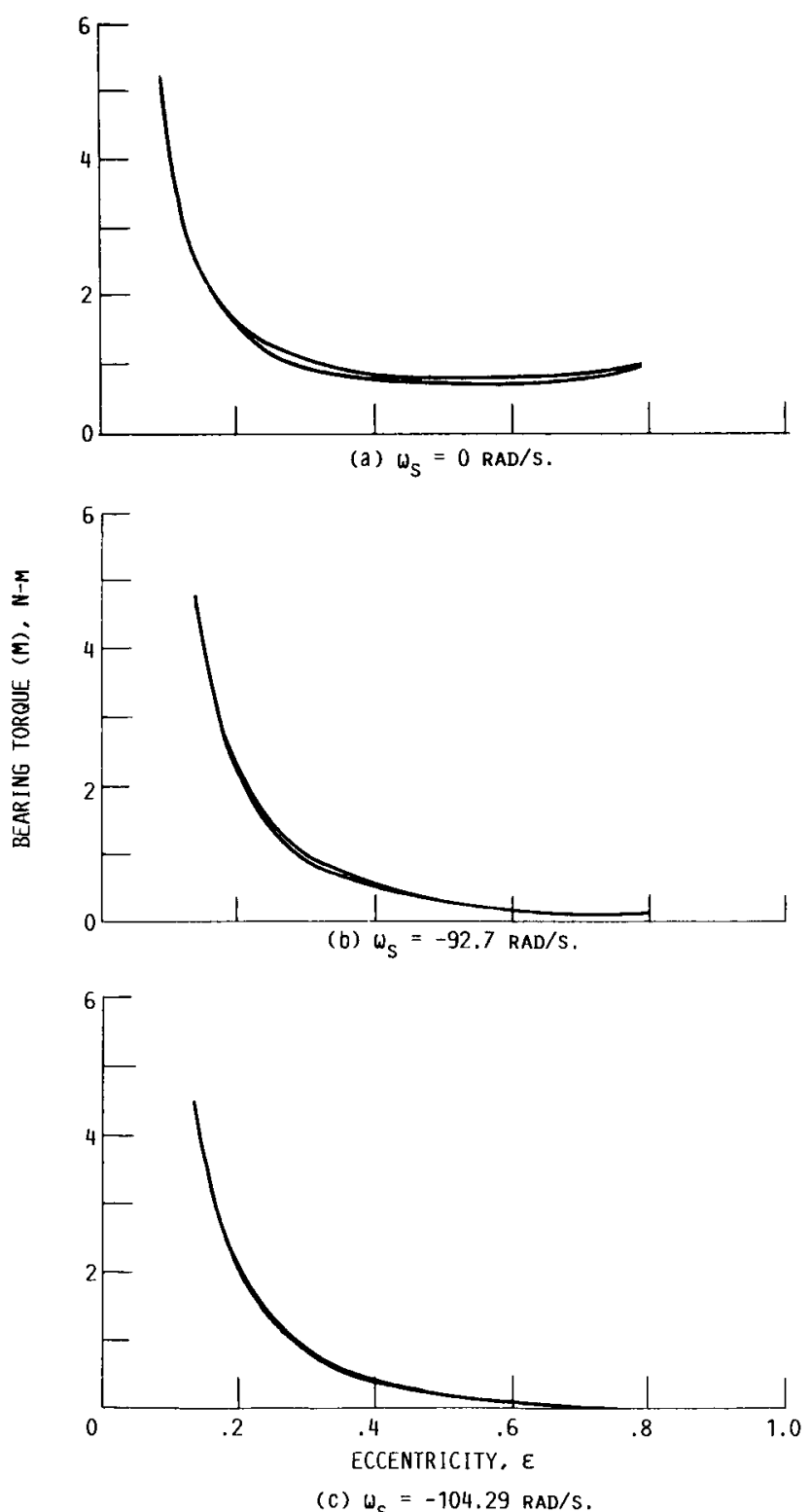

FI6. 8. - VARIATION OF BEARING TORQUE AS FUNCTION OF ECCENTRICITY. 


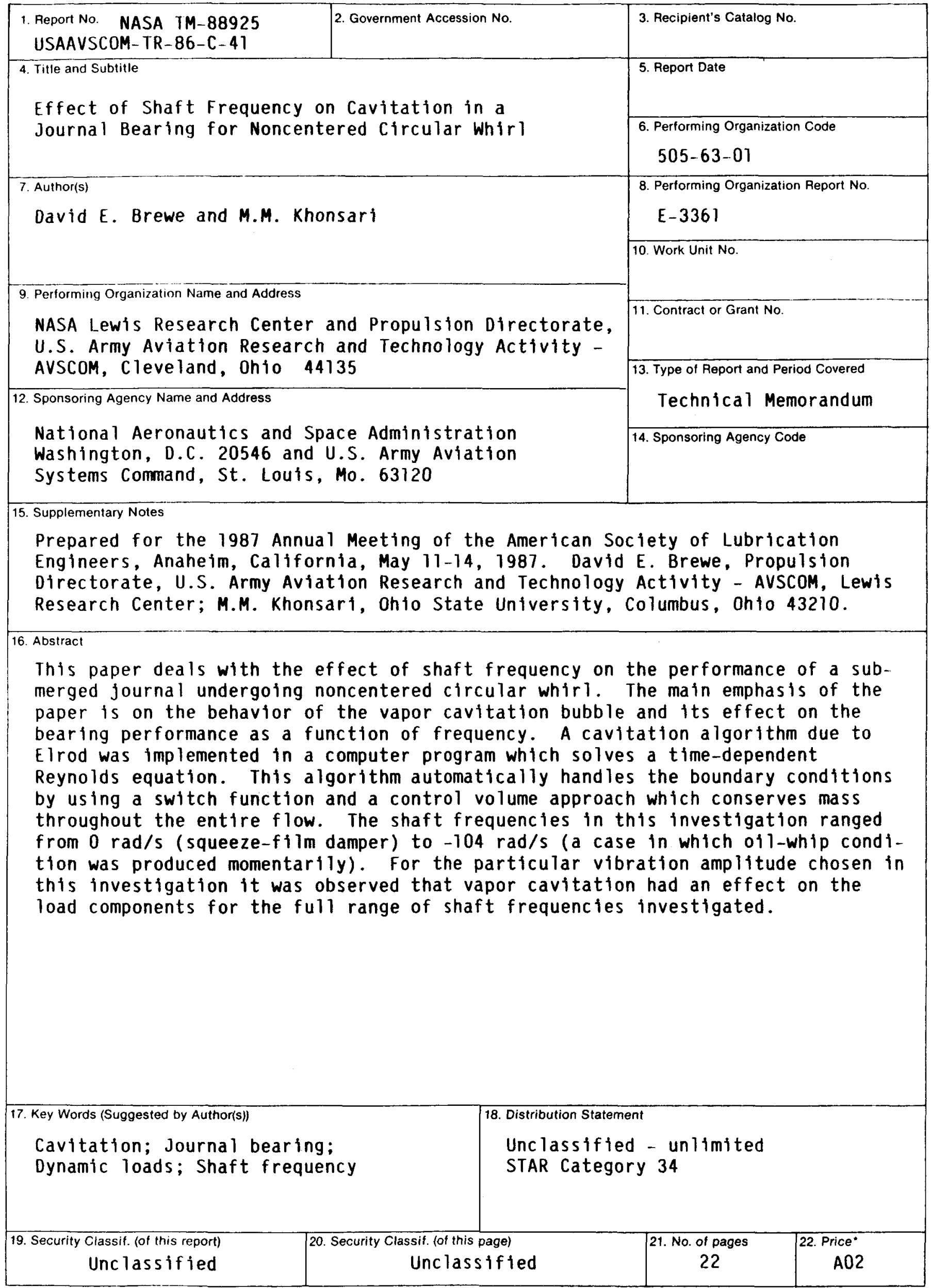

*For sale by the National Technical Information Service, Springfield, Virginia 22161 